\title{
Observer-Based Approach for Fractional-Order Chaotic Synchronization and Communication
}

\author{
Ibrahima N'Doye, Mohamed Darouach and Holger Voos
}

\begin{abstract}
This paper presents a method based on the state observer design for constructing a chaotically synchronized systems. Fractional-order direct Lyapunov theorem is used to derive the closed-loop asymptotic stability. The gains of the observer and observer-based controller are obtained in terms of linear matrix inequalities (LMIs) formulation. The proposed approach is then applied to secure communications. The method combines chaotic masking and chaotic modulation, where the information signal is injected into the transmitter and simultaneously transmitted to the receiver. Chaotic synchronization and chaotic communication are achieved simultaneously via a state observer design technique. An numerical fractional-order chaotic Lorenz system is given to demonstrate the applicability of the proposed approach.
\end{abstract}

Index Terms-Fractional-order calculus, fractional-order chaotic systems, fractional-order observer, linear matrix inequality (LMI), chaotic synchronization, secure communication, observer based controller.

\section{INTRODUCTION}

Over the past couple of decades, fractional calculus has gained significant attention as one of the topics that can be applied to a variety of fields in engineering. As is expected, there is no exception in controls regarding such an applied mathematics trend. A lot of studies and researches in controls have concentrated on using fractional calculus for modeling systems or designing controllers. Fractional-order systems (i.e. systems containing fractional derivatives and/or integrals) have been studied by many authors in engineering sciences from an application point of view (see [1], [2] and references therein). Many systems can be described with the help of fractional derivatives. These systems are known to display fractional-order dynamics : electromagnetic systems [3], dielectric polarization [4], viscoelastic systems [5].

The anomalous diffusion phenomena in inhomogeneous media can be explained by non-integer derivative based equations of diffusion [6]. Another example for an element with fractional-order model is the fractance. The fractance is an electrical circuit with non-integer order impedance [7]. This element has properties lie between resistance and capacitance. The resistance-capacitance-inductance (RLC) interconnect model of a transmission line is a fractionalorder model [8]. Heat conduction can be more adequately modeled by fractional-order models than by their integer

Ibrahima N'Doye and Holger Voos are with University of Luxembourg, Research Unit in Engineering Science (RUES), Faculté des Sciences, de la Technologie et de la Communication 6, rue Richard Coudenhove-Kalergi L-1359, Luxembourg.

Mohamed Darouach is with Université de Lorraine, Centre de Recherche en Automatique de Nancy (CRAN UMR-7039, CNRS), IUT de Longwy, 186 rue de Lorraine 54400 Cosnes et Romain, France. order counterparts [9]. In biology, it has been shown that the membranes of cells of biological organism have a fractionalorder electrical conductance [10]. In economics, it is known that some finance systems can display fractional order dynamics [11]. More recently, a lot of chaotic behaviors have been shown to be fractional-order systems [12], [13]. Then, controlling fractional-order systems becomes one of an active fields, especially controlling nonlinear fractionalorder systems. Also, fractional-order controller was used in [14] for flexible spacecraft attitude control.

The design of state estimators is one of the essential points in control theory whose solution, in the linear case, is the well known Luenberger's observer. Many contributions to the extension of the Luenberger observer for nonlinear systems has been proposed in the literature (see [15], [16], [17], [18] and references therein). The observer-based control is usually applied when we do not have access to all the states of a system. The notion of detectability which has been extended to nonlinear systems in [19], [20] allows to design nonlinear observer-based control using the separation principle.

In recent years chaos synchronization and chaotic secure communication have received ever increasing attention. Synchronization of chaotic systems has attracted noticeable interests and has been well studied for their potential applications in engineering sections, such as image processing, chemical and biological systems, information science, and secure communication [21], [22], [23], [24], [25].

The fractional-order derivative $\alpha_{i}$ imposes direct effect on the chaotic behavior of the nonlinear dynamical systems. Hence, it may also affect the synchronization (or control) performance of fractional-order systems. Some researchers have reported certain interesting results about the influence of fractional-order derivative $\alpha_{i}$ on the synchronization process. For example, the authors in [26] observed that the synchronization error decreases as the order $\alpha_{i}$ is increased. In other words, for larger value of $\alpha_{i}$ the synchronization starts earlier. A recent article [27] which studies the pinning control of fractional order complex network states show that it is much easier to control and synchronize a fractional order network with lower orders. A similar result is reported in [28], which indicates that smaller fractional-order can lead to better synchronization. The authors in [29] reveal that large coupling strengths and small fractional orders are both harmful for a successful identification and network topology.

An important application of chaotic synchronization is that the technique can be applied to secure communication easily. The secure communication system involves the development 
of a signal that contains the information that is to remain undetectable by interceptors within a carrier signal. We can ensure the security of this information by inserting it into a chaotic signal that is transmitted to a prescribed receiver that would be able to detect and recover the information from the chaotic signal.

To the best of our knowledge, [30], [31] are the two references that introduced designing observer based controller for nonlinear fractional-order systems. For the secure communication, a fractional chaotic communication method using an extended fractional Kalman filter is presented in [32].

In this paper, that may be seen as a generalization of the approach performed in [33], [34], we investigate generalized nonlinear fractional-order observers based approach for both chaos synchronization and secure communication. Results are established in the form of LMI, the observer gain could be found from the LMI formulation in the MATLAB environment.

The paper is organized as follows. In section II, we provide some background on the fractional derivative and the stability conditions for the linear fractional-order systems.

In section III, we give preliminary definitions and we consider the Lipschitz nonlinear fractional-order systems, the condition for the existence of the observer design problem for linear fractional-order systems are formulated.

In section IV, sufficient conditions for the convergence of the fractional-order state error dynamics are derived in terms of linear matrix inequalities formulation by using the fractional Lyapunov direct method.

In section $\mathrm{V}$, we propose a chaotic communication system using the state observer design method and sufficient conditions for the convergence of the fractional-order state error dynamics are derived in terms of linear matrix inequalities formulation by using the fractional Lyapunov direct method. Finally, an illustrative example is presented to illustrate our proposed results.

Notations. $\|x\|=\sqrt{x^{T} x}$ and $\|A\|=\sqrt{\lambda_{\max }\left(A^{T} A\right)}$ are the Euclidean vector norm and the spectral matrix norm respectively where $\lambda_{\max }\left(A^{T} A\right)$ is the maximal eigenvalue of the symmetric matrix $A^{T} A . M^{T}$ is the transpose of $M$ and $D^{\alpha}$ represents initialized $\alpha^{\text {th }}$ order differintegration. $\operatorname{spec}(A)$ is the set of all eigenvalues of $\mathrm{A}$.

\section{PRELIMINARY DEFINITIONS}

In this section, we first give the definition of fractionalorder fractional-order differentiation [1]. There are several forms of definitions of fractional derivative, such as Riemann-Liouville fractional derivative, Caputo's fractional derivative, Grünwald-Letnikov fractional derivative, and so on.

\section{A. Fractional derivative}

Fractional-order differentiation is the generalization of the integer-order ones. Formulations of noninteger-order derivatives fall into two main classes : the Riemann-Liouville derivative defined as [1]

${ }^{R} D^{\alpha} f(t)=\frac{1}{\Gamma(n-\alpha)} \frac{\mathrm{d}^{n}}{\mathrm{~d} t^{n}} \int_{0}^{t} \frac{f(\tau)}{(t-\tau)^{\alpha-n+1}} \mathrm{~d} \tau, n-1<\alpha<n$

or the Caputo derivative on the other, defined as [35],

$$
{ }^{C} D^{\alpha} f(t)=\frac{1}{\Gamma(\alpha-n)} \int_{0}^{t} \frac{\frac{\mathrm{d}^{n} f(\tau)}{\mathrm{d} t^{n}}}{(t-\tau)^{\alpha-n+1}} \mathrm{~d} \tau, n-1<\alpha<n
$$

with $n \in \mathbb{N}$ and $\alpha \in \mathbb{R}^{+}$, where $\Gamma($.$) is the Gamma function$ and is defined by the integral

$$
\Gamma(z)=\int_{0}^{\infty} e^{-t} t^{z-1} \mathrm{~d} t
$$

${ }^{R} D^{\alpha}$ and ${ }^{C} D^{\alpha}$ are Riemann-Liouville and Caputo fractional derivatives, respectively. The physical interpretation of the fractional derivatives and the solution of fractional differential equations are given in [35]. In this paper, the symbol $\mathcal{D}^{\alpha}$ is used to denote both the Caputo and Riemann-Liouville fractional derivative of order $\alpha$. For numerical simulation of the fractional-order systems, we can use the GrünwaldLetnikov method [36], [37] based on the Adams-BashforthMoulton type predictor-corrector scheme [38], [39]. The method is suitable for Caputo's derivative because it just requires the initial conditions and has a clear physical meaning for unknown functions. Due to the space limit, we do not present the detailed introduction of this numerical algorithm in this section, and the reader is referred to [38], [1], [36], [37] for more information.

Property 1: It is well known that Caputo's definition of fractional derivative is a modification of the RiemannLiouville definition and has the advantage of dealing with initial value problems in a proper way. Between the two definitions (Riemann-Liouville and Caputo fractional derivative), there are following relations [1]

${ }^{R} D^{\alpha} f(t)={ }^{C} D^{\alpha} f(t)+\sum_{k=0}^{n} \frac{t^{k-\alpha}}{\Gamma(k-\alpha+1)} f^{(k)}(0), n-1<\alpha<n$

${ }^{R} D^{\alpha}\left[f(t)-\sum_{k=0}^{n} f^{(k)}(0) \frac{t^{k}}{k !}\right]={ }^{C} D^{\alpha} f(t), n-1<\alpha<n$

Let us consider the Riemann-Liouville fractional derivative of order $\alpha$, then we have

$$
{ }^{R} D^{\alpha}(a)=\frac{a t^{-\alpha}}{\Gamma(1-\alpha)}
$$

where $a$ is a positive constant.

\section{B. Stability of fractional-order systems}

Lemma 1: [40] Let $x=0$ be an equilibrium point for the nonautonomous fractional-order system

$$
\mathcal{D}^{\alpha} x(t)=f(t, x) \quad 0<\alpha<1 .
$$

Assume that there exists a Lyapunov function $V(t, x(t))$ and class- $\mathcal{K}$ functions $\beta_{i}(i=1,2,3)$ satisfying

$$
\beta_{1}(\|x\|) \leqslant V(t, x) \leqslant \beta_{2}(\|x\|)
$$

and

$$
\mathcal{D}^{\alpha} V(t, x) \leqslant-\beta_{3}(\|x\|)
$$


Then the nonlinear fractional-order system (6) is asymptotically stable.

To proof the results in the section IV and $\mathrm{V}$, we need the following lemma.

Lemma 2: [41] Let $X$ and $Y$ be real vectors of the same dimension. Then, for any scalar $\varepsilon>0$, the following inequality holds

$$
X^{T} Y+Y^{T} X \leqslant \varepsilon X^{T} X+\varepsilon^{-1} Y^{T} Y .
$$

\section{PROBLEM FORMULATION}

Consider the following nonlinear fractional-order systems

$$
\left\{\begin{array}{l}
D^{\alpha} x(t)=A x(t)+f(x(t), y(t))+D d(t) \\
y(t)=C x(t)
\end{array} \quad 0<\alpha<1\right.
$$

where $x(t) \in \mathbb{R}^{n}$ is the state vector, $y(t) \in \mathbb{R}^{p}$ is the measured output and $d(t) \in \mathbb{R}^{q}$ is the unknown input vector. $A, B$ and $C$ are known constant real matrices with appropriate dimensions and $f(x(t), y(t))$ verify the Lipschitz condition, i.e., there exists $\lambda>0$ such that

$$
\|f(x, y)-f(\widehat{x}, y)\| \leqslant \lambda\|x-\widehat{x}\|
$$

for all $y(t) \in \mathbb{R}^{p}$ and $\widehat{x}(t) \in \mathbb{R}^{n}$ where $\lambda$ is a known Lipschitz constant

The necessary and sufficient conditions for the existence of the fractional-order observer for the linear case are given by the following lemma.

Lemma 3: [42], [43] The linear fractional-order system is detectable if and only

$$
\operatorname{rank}\left[\begin{array}{cc}
\sigma I_{n}-A & D \\
C & 0
\end{array}\right]=n \text { with }|\arg (\sigma)| \leqslant \alpha \frac{\pi}{2}, \forall \sigma \in \mathbb{C} .
$$

Remark 1: For the chaos synchronization, the chaotic drive system is modeled in (10) and the output $y(t)$ is used as an input to drive the chaotic response system, which is derived using the technique of observer design.

\section{ChaOtic SYNCHRONIZATION VIA AN OBSERVER DESIGN}

In this section, sufficient conditions for the convergence of the fractional-order state error dynamics are derived in terms of linear matrix inequalities formulation by using the fractional Lyapunov direct method.

Consider the following fractional-order observer for system (10)

$$
\left\{\begin{array}{l}
D^{\alpha} \widehat{x}(t)=A \widehat{x}(t)+f(\widehat{x}(t), y(t))+D d(t)+L(y(t)-\widehat{y}(t)) \\
\widehat{y}(t)=C \widehat{x}(t)
\end{array}\right.
$$

where $0<\alpha<1, \widehat{x}(t) \in \mathbb{R}^{n}$ is the estimate of $x(t)$ and $\widehat{y}(t) \in \mathbb{R}^{p}$ is the estimate output vector. Matrix $L$ is unknown matrix of appropriate dimension, which must be determined such that $\widehat{x}(t)$ asymptotically converges to $x(t)$.

Define $e(t)$, the error between $x(t)$ and $\widehat{x}(t)$

$$
e(t)=x(t)-\widehat{x}(t)
$$

then its dynamic is given by

$$
\mathcal{D}^{\alpha} e(t)=(A-L C) e(t)+f(x(t), y(t))-f(\widehat{x}(t), y(t))
$$

The asymptotical stability for the nonlinear fractionalorder observer error system (15) where $0<\alpha<1$ is given in the following theorem.

Theorem 1: Suppose that lemma 3 is satisfied (i.e. the pair $(C, A)$ is detectable), then there exists an asymptotically stable observer of the form (13) where $0<\alpha<1$, if there exist matrix $L$ and a positive scalar $\rho$ satisfying the following LMI

$$
(A-L C)^{T}+(A-L C)+\rho I<0 .
$$

where $\rho=\left(\varepsilon \lambda^{2}+2 \mu+\varepsilon^{-1}\right)$. didate

Proof: Consider the following Lyapunov function can-

$$
V(t)=2 e^{T}(t) e(t) .
$$

The fractional-order Caputo derivative of (17) is given by

${ }^{C} D^{\alpha} V(t)={ }^{R} D^{\alpha}\left(2 e^{T}(t) e(t)-\left[\sum_{k=0}^{n}\left(2 e^{T}(t) e(t)\right)^{(k)}(0) \frac{t^{k}}{k !}\right]_{\substack{k=0 \\(18)}}\right)$ or equivalently

$$
\begin{gathered}
{ }^{C} D^{\alpha} V(t)=\left({ }^{R} D^{\alpha} e^{T}(t)\right) e(t)+e^{T}(t)\left({ }^{R} D^{\alpha} e(t)\right) \\
-{ }^{R} D^{\alpha}\left(2 e^{T}(0) e(0)\right) \\
+2 \sum_{k=1}^{\infty} \frac{\Gamma(1+\alpha)}{\Gamma(1+k) \Gamma(1-k+\alpha)}{ }^{R} D^{k} e(t)^{R} D^{\alpha-k} e(t) .
\end{gathered}
$$

Using equation (5), equation (19) can be written as follows

$$
\begin{gathered}
{ }^{C} D^{\alpha} V(t)=\left({ }^{R} D^{\alpha} e^{T}(t)\right) e(t)+e^{T}(t)\left({ }^{R} D^{\alpha} e(t)\right) \\
-2 \frac{t^{-\alpha}}{\Gamma(1-\alpha)}\left(e^{T}(0) e(0)\right) \\
+2 \sum_{k=1}^{\infty} \frac{\Gamma(1+\alpha)}{\Gamma(1+k) \Gamma(1-k+\alpha)}{ }^{R} D^{k} e(t)^{R} D^{\alpha-k} e(t) .
\end{gathered}
$$

Using Caputo fractional derivative we obtain the following equation

$$
\begin{aligned}
{ }^{C} D^{\alpha} V(t)=\left({ }^{C} D^{\alpha} e^{T}(t)\right) e(t)+e^{T}(t)\left({ }^{C} D^{\alpha} e(t)\right) \\
-2 \frac{t^{-\alpha}}{\Gamma(1-\alpha)}\|e(0)\|^{2}+2 \Upsilon_{e}(t),
\end{aligned}
$$

where

$$
\Upsilon_{e}(t)=\sum_{k=1}^{\infty} \frac{\Gamma(1+\alpha)}{\Gamma(1+k) \Gamma(1-k+\alpha)}{ }^{C} D^{k} e(t)^{C} D^{\alpha-k} e(t)
$$

and we can consider the following boundedness condition

$$
\Upsilon_{e}(t) \leqslant \mu\|e(t)\|^{2} \text {. }
$$

Since $2 \frac{t^{-\alpha}}{\Gamma(1-\alpha)}\|e(0)\|^{2} \geqslant 0$ and substituting (15) into (21), one can easily conclude that

$$
\begin{gathered}
{ }^{C} D^{\alpha} V \leqslant e^{T}(t)\left((A-L C)^{T}+(A-L C)\right) e(t)+e^{T}(t) \delta(t) \\
+\delta^{T}(t) e(t)+2 \Upsilon_{e}(t),
\end{gathered}
$$

where $\delta(t)=f(\widehat{x}(t))-f(x(t))$.

By using the relation (9), we obtain the following inequality

$$
e^{T}(t) \delta(t)+\delta^{T}(t) e(t) \leqslant \varepsilon \delta^{T}(t) \delta(t)+\varepsilon^{-1} e^{T}(t) e(t)
$$

and from the Liptschitz constraint we have

$$
\delta^{T}(t) \delta(t) \leqslant \lambda^{2}\|\widehat{x}(t)-x(t)\|^{2}=\lambda^{2} e^{T}(t) e(t) .
$$

Then we obtain

$$
{ }^{C} D^{\alpha} V \leqslant e^{T}(t)\left[(A-L C)^{T}+(A-L C)+\rho I\right] e(t) .
$$


where $\rho=\left(\varepsilon \lambda^{2}+2 \mu+\varepsilon^{-1}\right)$.

Using the fractional direct Lyapunov method in lemma 1, the sufficient conditions for asymptotically stability of the fractional-order state error dynamics $e(t)$ is choosing $L$ and $\rho$ such that

$$
(A-L C)^{T}+(A-L C)+\rho I<0 .
$$

This ends the proof.

Lemma 4: The inequality in (28) is satisfied if the pair $(C, A)$ is observable [43] and the following condition is satisfied [18]

$$
\min \rho_{\min }\left(A-L C-\sigma I_{3}\right)>\lambda_{\max }, \quad \forall \sigma \in \mathbb{C} .
$$

where $\rho_{\text {min }}$ denotes the minimum singular value of a matrix. If the pair $(C, A)$ is observable, then the gain matrix $L$ can be selected so that [42]

$$
|\arg (\operatorname{spec}(A-L C))|>\alpha \frac{\pi}{2} .
$$

Since $\operatorname{rank}(C D)=\operatorname{rank} D=q$ the condition

$$
\operatorname{rank}\left[\begin{array}{cc}
\sigma I_{n}-A & D \\
C & 0
\end{array}\right]=n+q \text { with }|\arg (\sigma)| \leqslant \alpha \frac{\pi}{2}, \forall \sigma \in \mathbb{C}
$$

implies that the pair $(C, A)$ is observable [43]. Then, the matrix $A$ in (10) should be chosen such that the condition in (31) is satisfied.

\section{Chaotic SeCURE COMMUNiCATION Via AN OBSERVER DESIGN}

In this section, we propose a chaotic communication system using the state observer design method. The proposed communication system consist of a transmitter and a receiver at the receiving end of communication. The transmitter is a chaotic system described by (10) with a slight modification and represented as follows

$$
\left\{\begin{array}{l}
D^{\alpha} x(t)=A x(t)+f(x(t), \bar{y}(t))+B d(t)+L s(t) \\
\bar{y}(t)=C x(t)+s(t) \\
x(0)=x_{0}
\end{array}\right.
$$

where $0<\alpha<1, s(t) \in \mathbb{R}^{p}$ is the information signal and $\bar{y}(t) \in \mathbb{R}^{p}$ is the chaotically transmitted signal which drives the receiver. Using the state observer design, the receiver is then constructed as follows

$$
\left\{\begin{array}{l}
D^{\alpha} \widehat{x}(t)=A \widehat{x}(t)+f(\widehat{x}(t), \bar{y}(t))+B d(t)+L(\bar{y}(t)-\widehat{y}(t)) \\
\widehat{y}(t)=C \widehat{x}(t) \\
\widehat{x}(0)=x_{0}
\end{array}\right.
$$

where $0<\alpha<1$ and matrix $L$ is unknown matrix of appropriate dimension, which must be determined such that $\widehat{x}(t)$ asymptotically converges to $x(t)$.

Similarly, define the synchronization error $e(t)$, the error between $x(t)$ and $\widehat{x}(t)$

$$
e(t)=x(t)-\widehat{x}(t)
$$

then its dynamic is given by

$$
\mathcal{D}^{\alpha} e(t)=(A-L C) e(t)+f(x(t), \bar{y}(t))-f(\widehat{x}(t), \bar{y}(t))
$$

Moreover, the function $f(x(t), \bar{y}(t))$ satisfies the Lipschitz condition i.e., there exists $\bar{\lambda}>0$ such that

$$
\|f(x, \bar{y})-f(\widehat{x}, \bar{y})\| \leqslant \bar{\lambda}\|x-\widehat{x}\|
$$

for all $\bar{y}(t) \in \mathbb{R}^{p}$ and $\widehat{x}(t) \in \mathbb{R}^{n}$ where $\bar{\lambda}$ is a known Lipschitz constant

The asymptotical stability for the nonlinear fractionalorder synchronization error system (35) where $0<\alpha<1$ is given in the following theorem.

Theorem 2: Assume that lemma 3 is satisfied (i.e. the pair $(C, A)$ is detectable), then there exists an asymptotically stable observer of the form (33) where $0<\alpha \leqslant 1$, if there exist matrix $L$ and a positive scalar $\bar{\rho}$ satisfying the following LMI

$$
(A-L C)^{T}+(A-L C)+\bar{\rho} I<0 .
$$

where $\bar{\rho}=\left(\varepsilon \bar{\lambda}^{2}+2 \mu+\varepsilon^{-1}\right)$.

Proof: The proof of this theorem is similarly to the proof of theorem 1.

The recovered signal is achieved by

$$
s_{R}(t)=\bar{y}(t)-\widehat{y}(t)
$$

and using the previous results, we have

$$
\lim _{t \rightarrow \infty} s_{R}(t)=\lim _{t \rightarrow \infty}(C e(t)+s(t))=s(t) .
$$

Consequently, the information signal can be asymptotically recovered at the receiving end of communication.

Remark 2: For secure communications applications, the transmitted signal is a sum of the information and the output of the chaotic transmitter. In addition, the transmitted signal is also injected into the transmitter and, simultaneously, transmitted to the receiver. By the observer design technique, a chaotic receiver is then derived to recover the information signal at the receiving end of the communication. In the conventional chaotic masking method, the receiver is driven by the sum of the information signal and the output of the transmitter, whose dynamics are autonomous. However, in the communication system proposed herein, the transmitted signal is also fed back into the transmitter, exactly like that of [44], [34]. Hence, the dynamics of the transmitter are driven by a time-varying signal $\bar{y}(t)$, thereby implying that the transmitter is a nonautonomous system, which is generally more complicated.

\section{NUMERICAL EXAMPLE}

In this section, we provide an numerical example to illustrate the chaotic synchronization and secure communication scheme proposed herein, the chaotic Lorenz system is considered and the numerical simulation is performed.

Consider the following fractional-order chaotic Lorenz system where integer-order derivatives are replaced by fractional-order ones. Mathematical description of the fractional-order chaotic system is expressed as

$$
\left\{\begin{array}{l}
D^{\alpha} x=\left[\begin{array}{ccc}
-10 & 10 & 0 \\
28 & -1 & 0 \\
0 & 0 & -\frac{8}{3}
\end{array}\right] x+\left[\begin{array}{c}
0 \\
-x_{1} x_{3} \\
x_{1} x_{2}
\end{array}\right] \quad 0<\alpha<1 \\
y=\left[\begin{array}{lll}
1 & 0 & 0
\end{array}\right] x
\end{array}\right.
$$


where $x=\left[\begin{array}{lll}x_{1} & x_{2} & x_{3}\end{array}\right]^{T}$.

We find that chaos exists in the fractional-order Lorenz system (40). The necessary condition to exhibit double-scroll attractor of system (40) is the eigenvalues $\lambda_{1,2}=\omega_{1,2} \pm \beta_{1,2}$ remaining in the unstable region [45], [37]. The condition for commensurate derivatives order is

$$
\alpha>\frac{2}{\pi} \arctan \left(\frac{\left|\beta_{i}\right|}{\omega_{i}}\right) \quad \text { with } \quad i=1,2 .
$$

This condition can be used to determine the minimum order for which a nonlinear system can generate chaos [46], [37]. The minimal order for which the Lorenz system is chaotic is given in relation (41). In this case the minimal commensurate order is $\alpha>0.9941$ where $\alpha_{1}=\alpha_{2}=\alpha_{3}=\alpha$.

Let us set $\alpha_{1}=\alpha_{2}=\alpha_{3}=\alpha=0.995$ in our example, the fractional-order Lorenz's system (40) has a chaotic attractor as depicted in Fig. 1 where the initial conditions set to $x_{0}=$ $\left[\begin{array}{lll}2 & 2 & 2\end{array}\right]^{T}$.

The transmitter for chaotic communication is given by

$$
\left\{\begin{array}{l}
D^{\alpha} x=\left[\begin{array}{ccc}
-10 & 10 & 0 \\
28 & -1 & 0 \\
0 & 0 & -\frac{8}{3}
\end{array}\right] x+\left[\begin{array}{c}
0 \\
-\bar{y} x_{3} \\
\bar{y} x_{2}
\end{array}\right]+L s \\
\bar{y}=\left[\begin{array}{lll}
1 & 0 & 0
\end{array}\right] x+s
\end{array}\right.
$$

where $0<\alpha<1$ and $s(t)$ is the information signal. The receiver based on a state observer is designed as follows

$$
\left\{\begin{array}{l}
D^{\alpha} \widehat{x}=\left[\begin{array}{ccc}
-10 & 10 & 0 \\
28 & -1 & 0 \\
0 & 0 & -\frac{8}{3}
\end{array}\right] \widehat{x}+\left[\begin{array}{c}
0 \\
-\bar{y}(t) \widehat{x}_{3} \\
\bar{y}(t) \widehat{x}_{2}
\end{array}\right]+L(\bar{y}-\widehat{y}) \\
\widehat{y}=\left[\begin{array}{lll}
1 & 0 & 0
\end{array}\right] \widehat{x}
\end{array}\right.
$$

For the numerical simulation, the information signal $s(t)$ is chosen as a sinusoidal signal i.e., $s(t)=0.05 \sin (60 \pi \times t)$.

From the results of section $\mathrm{V}$, a feasible solution of LMI (37) is as follows

$$
L=\left[\begin{array}{lll}
-8.1368 & 38 & 0
\end{array}\right] .
$$

The linear part of the system described in (40) is detectable for the fractional-order derivatives $\alpha=0.995$. The simulation results are presented in Figs. 2, 3, 4 and 5 with the fractional-order derivative $\alpha=0.995$. The initial conditions are set to $x_{0}=\left[\begin{array}{lll}2 & 2 & 2\end{array}\right]^{T}$ while the initial values of their estimates are $\widehat{x}_{0}=\left[\begin{array}{lll}10 & 10 & 10\end{array}\right]^{T}$. From the simulations results, it can be seen that the zero estimation errors have been achieved, which implies that the proposed observer in this paper works effectively.

\section{CONCLUSION}

In this paper, a fractional-order observer-based approach has been presented to resolve the chaotic synchronization of a class of chaotic systems and its application to secure communications. Sufficient conditions for the asymptotical stability of the state error dynamics are derived in terms of linear matrix inequalities LMIs formulation and fractional-

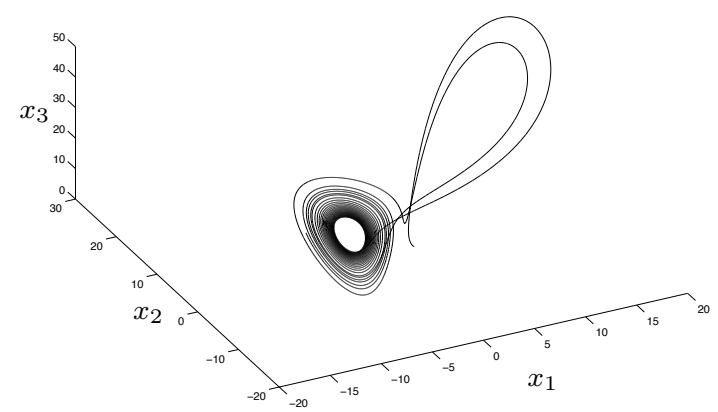

Fig. 1. Chaotic attractors in the fractional-order Lorenz chaotic system with $\alpha=0.995$

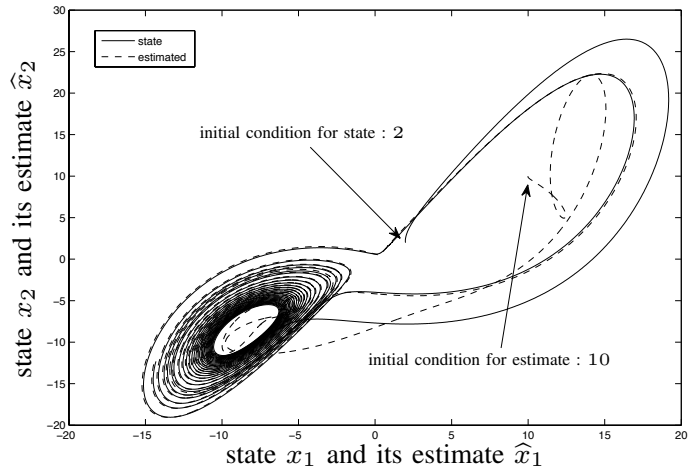

Fig. 2. Phase portrait of $x_{1}$ versus $x_{2}$ (solid lines) and $\widehat{x}_{1}$ versus $\widehat{x}_{2}$ (dashed lines) with $\alpha=0.995$

order direct Lyapunov theorem with fractional-order belonging to $0<\alpha<1$. Performances of the proposed method have been shown through the Lorenz chaotic model.

\section{REFERENCES}

[1] I. Podlubny, Fractional Differential Equations. New York: Academic, 1999.

[2] R. Hilfer, Applications of Fractional Calculus in Physics. Singapore: World Scientific Publishing, 2001.

[3] N. Engheta, "On fractional calculus and fractional multipoles in electromagnetism," IEEE Trans. Antennas and Propagation, vol. 44, pp. 554-566, 1996.

[4] H. Sun, A. Abdelwahad, and B. Onaral, "Linear approximation of transfer function with a pole of fractional order," IEEE Trans. Aut. Contr, vol. 29, pp. 441-444, 1984.

[5] R. Bagley and R. Calico, "Fractional order state equations for the control of viscoelastically damped structures," J. Guidance, Contr. \& Dynamics, vol. 14, pp. 304-311, 1991.

[6] L. El Ghaoui and G. Scorletti, "Control of rational systems using linear-fractional representations and linear matrix inequalities," Automatica, vol. 32, pp. 1273-1284, 1996.

[7] A. Le Méhauté and G. Crepy, "Introduction to transfer and motion in fractal media: The geometry of kinetics," Solid State Ionics, vol. 9-10, pp. 311-322, 1983.

[8] G. Chen and E. Friedman, "An RLC interconnect model based on Fourier analysis," IEEE Trans. Computer-Aided Design of Integrated Circuits and Systems, vol. 24, pp. 170-183, 2005.

[9] V. Jenson and G. Jeffreys, Mathematical Methods in Chemical Engineering. New York: Academic Press, 1977.

[10] K. Cole, "Electric conductance of biological systems," in Proc. Cold Spring Harbor Symp. Quantitative Biology, (New York, USA), pp. 107-116, 1933.

[11] N. Laskin, "Fractional market dynamics," Physica A: Statistical Mechanics and its Applications, vol. 287, pp. 482-492, 2000.

[12] C. Li and G. Chen, "Chaos in the fractional order Chen system and its control," Chaos, Solitons \& Fractals, vol. 22, pp. 549-554, 2004.

[13] I. Petrás̆, "A note on the fractional-order Chua's system," Chaos, Solitons \& Fractals, vol. 38, pp. 140-147, 2008. 


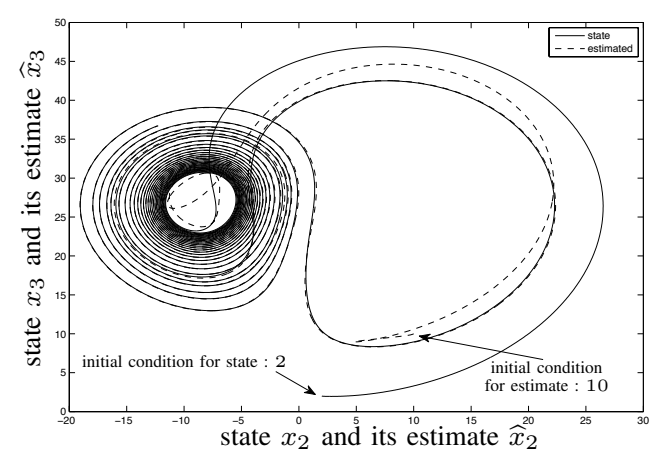

Fig. 3. Phase portrait of $x_{2}$ versus $x_{3}$ (solid lines) and $\widehat{x}_{2}$ versus $\widehat{x}_{3}$ (dashed lines) with $\alpha=0.995$

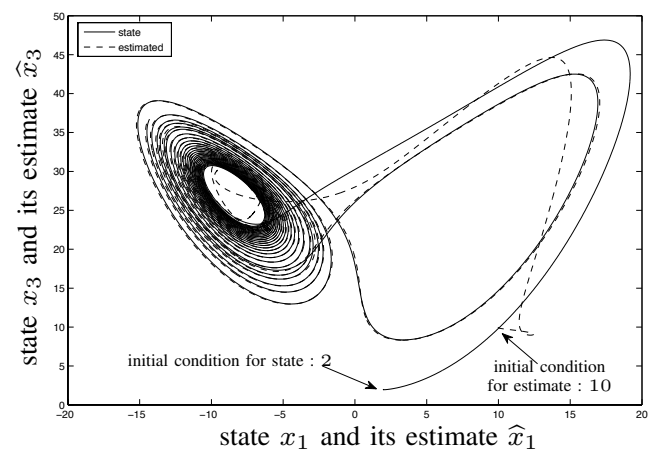

Fig. 4. Phase portrait of $x_{1}$ versus $x_{3}$ (solid lines) and $\widehat{x}_{1}$ versus $\widehat{x}_{3}$ (dashed lines) with $\alpha=0.995$

[14] S. Manabe, "A suggestion of fractional-order controller for flexible spacecraft attitude control," Nonlinear Dynamics, vol. 29, pp. 251268, 2002.

[15] J. Tsinias, "Observer design for nonlinear systems," Syst. \& Contr. Letters, vol. 13, pp. 135-142, 1989.

[16] J. Gauthier, H. Hammouri, and S. Othman, "A simple observer for nonlinear systems, applications to bioreactors," IEEE Trans. Aut. Contr., vol. 37, pp. 875-880, 1992.

[17] S. Raghavan and J. Hedrick, "Observer design for a class of nonlinear systems," Int. J. Contr., vol. 59, pp. 515-528, 1994.

[18] R. Rajamani, "Observer for Lipschitz nonlinear systems," IEEE Trans. Aut. Contr, vol. 43, pp. 397-401, 1998.

[19] E. Sontag and Y. Wang, "Output-to-state stability and detectability of nonlinear systems," Syst. \& Contr. Letters, vol. 29, pp. 279-290, 1997.

[20] J. Hespanha and A. Morse, "Certainty equivalence implies detectability," Syst. \& Contr. Letters, vol. 36, pp. 1-13, 1999.

[21] G. Chen and X. Dong, From Chaos to Order : Perspectives, Methodologies and Applications. Singapore: World scientific Publishing, 1998.

[22] A. Pikovsky, M. Rosenblum, and J. Kurths, Synchronization : A Universal Concept in Nonlinear Sciences. Cambridge: Cambridge University Press, 2001.

[23] S. K. Dana, P. K. Roy, and J. Kurths, Complex Dynamics in Physiological Systems : From Heart to Brain. New York: Springer, 2009.

[24] J. G. Ojalvo and R. Roy, "Spatiotemporal communication with synchronized optical chaos," Physical Review Letters, vol. 86, pp. 52045207, 2001.

[25] J. C. Sprott, Chaos and Time-Serie Analysis. Oxford: Oxford University Press, 2003.

[26] S. Bhalekar and V. Daftardar-Gejji, "Synchronization of different fractional-order chaotic systems using active control," Commun Nonlinear Sci. Numer. Simulat., vol. 15, pp. 3536-3546, 2010.

[27] Y. Tang, Z. Wang, and J. Fang, "Pinning control of fractional-order weighted complex networks," Chaos, vol. 19, p. 013112, 2009.

[28] X. Wu, D. Lai, and H. Lu, "Generalized synchronization of the fractional-order chaos in weighted complex dynamical networks with nonidentical nodes," Nonlinear Dynamics, vol. 69, pp. 667-683, 2012.

[29] G. Si, Z. Sun, H. Zhang, and Y. Zhang, "Parameter estimation and topology identification of uncertain fractional order complex

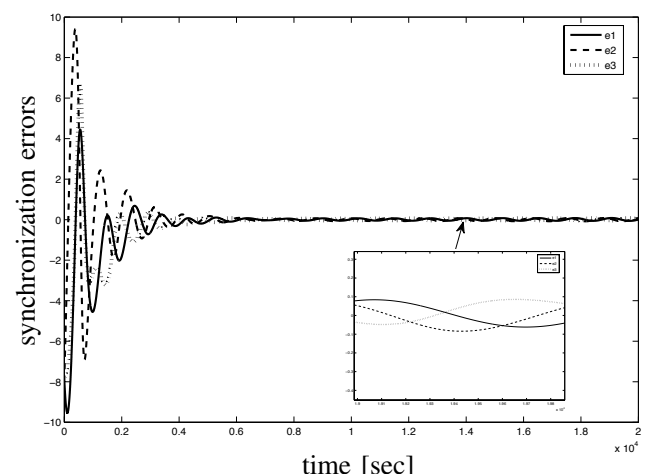

Fig. 5. Synchronization errors $e_{1}=x_{1}-\widehat{x}_{1}, e_{2}=x_{2}-\widehat{x}_{2}$ and $e_{3}=$ $x_{3}-\widehat{x}_{3}$ with $\alpha=0.995$

networks," Commun Nonlinear Sci. Numer. Simulat., vol. 17, pp. 5158$5171,2012$.

[30] I. N'Doye, M. Zasadzinski, M. Darouach, and N. Radhy, "Observerbased control for fractional-order continuous-time systems," in Proc. IEEE Conf. Decision \& Contr., (Shanghai, P.R. China), 2009.

[31] E. A. Boroujeni and H. R. Momeni, "Observer based control of a class of nonlinear fractional-order systems using LMI," International Journal of Science and Engineering Investigations, vol. 1, pp. 48-52, 2012.

[32] A. Kiani-B, K. Fallahi, N. Pariz, and H. Leung, "A chaotic secure communication scheme using fractional chaotic systems based on an extended fractional kalman filter," Nonlinear Science and Numerical Simulation, vol. 14, pp. 863-879, 2007.

[33] M. Boutayeb, M. Darouach, and H. Rafaralahy, "Generalized statespace observers for chaotic synchronization and secure communication," IEEE Trans. Circ. Syst. I : Fund. Theory \& Appli., vol. 49, pp. 345-349, 2002.

[34] T. Liao and N. Huang, "An observer-based approach for chaotic synchronization with applications to secure communications," IEEE Trans. Circ. Syst. I : Fund. Theory \& Appli., vol. 46, pp. 1144-1150, 1999.

[35] I. Podlubny, "Geometric and physical interpretation of fractional integration and fractional differentiation," Fractional Calculus \& Applied Analysis, vol. 5, pp. 367-386, 2002.

[36] I. Petrášs, "A note on the fractional-order Volta system," Commun Nonlinear Sci. Numer. Simulat., vol. 15, pp. 384-393, 2010.

[37] I. Petráš, Fractional-Order Nonlinear Systems : Modeling, Analysis and Simulation. Berlin: Springer, 2011.

[38] L. Dorckák, "Numerical models for simulation the fractional-order control systems," Tech. Rep. UEF-04-94, Institute of Experimental Physics, Academy of Sciences, Slovakia, 1994.

[39] W. Deng, "Short memory principle and a predictor-corrector approach for fractional differential equations," J. Comput. Appl. Math., vol. 206, pp. 174-188, 2007.

[40] Y. Li, Y. Chen, and I. Podlubny, "Stability of fractional-order nonlinear dynamic systems : Lyapunov direct method and generalized Mittag-Leffler stability," Computers \& Mathematics with Applications, vol. 59, pp. 1810-1821, 2010.

[41] P. Khargonakar, I. Petersen, and K. Zhou, "Robust stabilization of uncertain linear systems : quadratic stability and $\mathcal{H}_{\infty}$ control theory," IEEE Trans. Aut. Contr., vol. 35, pp. 356-361, 1990.

[42] D. Matignon and B. Andréa-Novel, "Observer-based for fractional differential systems," in Proc. IEEE Conf. Decision \& Contr., (San Diego, USA), 1997.

[43] M. Darouach, M. Zasadzinski, and S. Xu, "Full-order observers for linear systems with unknown inputs," IEEE Trans. Aut. Contr., vol. 39, pp. 606-609, 1994.

[44] V. Milanovic and M. E. Zaghloul, "Improved masking algorithm for chaotic communication systems," Electron Letters, vol. 32, pp. 11-12, 1996.

[45] S. M. Tavazoei and M. Haeri, "Limitations of frequency domain approximation for detecting chaos in fractional order systems," Nonlinear Analysis, vol. 69, pp. 1299-1320, 2008.

[46] S. M. Tavazoei and M. Haeri, "A necessary condition for double scroll attractor existence in fractional-order systems," Physics Letters A, vol. 367, pp. 102-113, 2007. 